\title{
WANN HAT DAS FEST BEI ADRASTOS STATTGEFUNDEN? EINE UNTERSUCHUNG ZU STAT. THEB. 1. 692-693
}

\begin{abstract}
Summary: Astronomical phenomena play a specific role in ancient literature to illustrate the internal chronology of the plot. It is obvious that especially poetical texts which deal with constellations and astronomical terms show a maximum of the so called poetical doctrina as - for example - can be seen in the work of Statius. The present paper thus tries to prove this thesis by analyzing the verses 692-693 of the first book of the Thebais. Therein the vanishing of the Great Bear/Big Dipper constellation seems to refer to a specific season.
\end{abstract}

Key words: Statius, Thebais, astronomical phenomena, constellation, Big Dipper, Great Bear

Otta Wenskus schreibt in der Einleitung zu ihrer Abhandlung „Astronomische Zeitangaben von Homer bis Theophrast“ über die Zielsetzung der 1990 publizierten Arbeit das Folgende: ${ }^{1}$ „Die meisten Astronomiehistoriker haben sich (...) nicht oder nur am Rande mit den Problemen beschäftigt, die im Mittelpunkt der vorliegenden Arbeit stehen: diese beschäftigt sich weniger mit einem Teilgebiet der Wissenschaftsgeschichte als mit Angaben, die oft nur auf den ersten Blick präzise sind und sich auf literarische Klischees oder populäre Verallgemeinerungen zurückführen lassen.“ Solche Angaben wiederum enthielten ,(...) nicht selten Sachaussagen, deren Sinn bisher verkannt worden ist (...)“, zumal es „,...) keine literarische Gattung innerhalb der griechischen Literatur gibt, die frei von solchen astronomischen Zeitangaben wäre“.

Dass sich diese Aussagen auf die lateinische Literatur transferieren lassen, ist sicher nicht zu leugnen. So finden sich bereits seit Vergil in den Epen - Ovids Fasti wei-

${ }^{1}$ WenSKus, O.: Astronomische Zeitangaben von Homer bis Theophrast. Stuttgart 1990, 9. 
sen eine Vielzahl solcher Beispiele auf ${ }^{2}$ - astronomische Zeitangaben, ${ }^{3}$ deren Tradition bis zu den Werken Homers zurückreicht. Der Vermutung, dies hätte allein topischen Charakter, sollte entgegengehalten werden, dass bestimmte Verweise auf Sternkonstellationen und deren Auf- bzw. Untergang wohl nicht gänzlich wahllos und willkürlich gesetzt worden sind. Schon der Redelehrer Quintilian spricht von einer gewissen Notwendigkeit astronomischen Wissens bei der Lektüre von Dichtung, da Dichter häufig die Zeit nach dem Auf- und Untergehen von Sternen spezifiziert haben. ${ }^{4}$

$\mathrm{Ob}$ zu solchen poetae auch der Epiker Statius zu zählen sein sollte, dem diese kurze Studie gewidmet ist, möchte ich exemplarisch an einer Stelle aus dem ersten Buch der Thebais untersuchen.

\section{GLIEDERUNG DES ERSTEN BUCHES DER THEBAIS UND FRAGESTELLUNG}

Das erste Buch der Thebais gliedert sich in zwei große Teile. Nach einem 45 Verse umfassenden Prolog, der das obligatorische Enkomium auf den Kaiser und den Musenanruf enthält, beginnt Statius die eigentliche Haupthandlung mit dem Fluch des Ödipus (46-87), woran sich Tisiphones Weg nach Theben (88-123) und ihr Wirken auf die Brüder Eteocles und Polyneices (123-164) anschließen. In einer Art Rekurs wird das Verhalten der Thebaner zum Herrschaftspakt der Brüder dargestellt, an dessen Ende eine Götterversammlung auf dem Olymp steht (197-311). Der zweite Teil des ersten Buches behandelt die Ereignisse um Polyneices und Tydeus in Argos. Polyneices, der sich auf dem Weg nach Argos befindet (312-389), erreicht die Stadt etwa zeitgleich mit Tydeus, der von Calydon nach Argos kommt (390-407). Es entsteht ein heftiger und gewalttätiger Streit der beiden Heroen (408-427), der durch den vom Lärm aufgeschreckten Adrastos geschlichtet wird (428-481). Die Helden betreten nun den Palast (482-510) und nehmen an dem dort stattfindenden Gastmahl teil (510-720), während selbigem König Adrastos den Mythos von Coroebus erzählt (557-667), nach der Abstammung der beiden Helden fragt (668-672) und milde auf Polyneices' Bekenntnis

\footnotetext{
${ }^{2}$ Vgl. dazu jüngst GEE, E.: Ovid, Aratus and Augustus: Astronomy in Ovid's Fasti. Cambridge 2008, 9-65, 205-208.

${ }^{3}$ Vergil verweist beispielsweise in der Aeneis an zwei Stellen auf den Arktur und den Aufgang der Hyaden (Aen. 1. 744; 3. 516). Ebenso nennt er an diesen Stellen die beiden Bären (Trionen). Lucan wiederum nennt den Arktophylax (8. 180) bzw. Bootes (2. 722; 3. 252; 10. 289) namentlich. Auch Helice (2. 237) und Kynosura (3. 219; 8. 180; 9. 540) werden von ihm erwähnt; vgl. dazu BoLL, F. - GuNDEL, W.: Sternbilder, Sternglaube und Sternsymbolik bei Griechen und Römern. In Ausführliches Lexikon der griechischen und römischen Mythologie von W. H. ROSCHER. Bd. 6. Leipzig-Berlin 1924-1937 [Nachdruck Hildesheim - New York 1965 und Hildesheim 1977], 867-1071; GUNDEL, W.: Kynosura (6). In RE XII 1 (1924) 37-41; GundeL, H. G.: Ursa. In RE IX A (1961) 1033-1054; LE BUEFFLE, A.: Les noms latins d'astres et de constellations. Paris 1977; MONTANARI CALDINI, R.: Le stelle dell' Orsa Maggiore (Septem Triones) negli Aratea di Cicerone. In SAntini, C. - Zurli, L. - CARDinAli, L. (a cura di): Concentus ex dissonis. Scritti in onore di Aldo Setaioli. Vol. I. Napoli 2006, 123-136; bes. 125 mit Bibliographie.

${ }^{4}$ Quint. inst. 1. 4. 4: nec si rationem siderum ignoret poetas [grammatice] intellegat, qui, ut alia mittam, totiens ortu occasuque signorum in declarandibus temporibus utuntur (...).
} 
(673-681) reagiert (681-692). Dass die Zeit nun gekommen sei, den Päan anzustimmen (694-720), gibt Adrastos in folgender interessanten Wendung zu verstehen, in der auf das Untergehen eines bestimmten Sternbildes hingewiesen wird (692-693):

Sed iam temone supino

languet Hyperboreae glacialis portitor Ursae.

Doch schon ermattet der eisige Träger

des hyperboräischen Bären

mit zurückgebogener Deichsel.

Aus diesen astronomischen Angaben zum Untergang des Sternbildes Großer Bär stellen sich nun zwei durchaus interessante Fragenkomplexe. Erstens: Konnte Statius bei der Konzeption der Stelle auf literarische Vorbilder zurückgreifen oder waren seine Angaben unbeeinflusst? Zweitens: Ist es möglich, aus den Angaben des Dichters das von Adrastos in Statius' Fiktion ausgerichtete Gastmahl zeitlich in eine bestimmte Jahreszeit einzuordnen?

Um diese Fragen zu beantworten, bietet es sich an, zuerst nach Vorbildern zu suchen und in einem zweiten Schritt das Erscheinen der angesprochenen Sternkonstellation zur Zeit der Abfassung im ersten nachchristlichen Jahrhundert näher zu betrachten.

\section{VORBILDER}

\section{Griechische Vorbilder}

Dass das Sternbild des (Großen) Bären und mit ihm das des Bärenhüters Bootes von Alters her bekannt waren, zeigen verschiedene Zeugnisse der griechischen Literatur, deren Bekannteste an dieser Stelle genannt seien.

Die Bezeichnung des erwähnten Sternbildes als Bootes, Bär und Wagen erscheint erstmals bei Homer in Od. 5. 271-275. ${ }^{5}$ Auffällig an dieser Stelle ist der Hinweis, dass

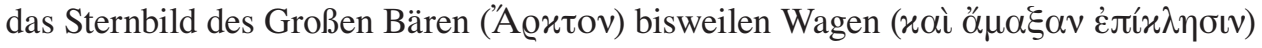
genannt wird.

Auch in der Ilias ist der Große Bär unter verschiedenen Bezeichnungen bekannt. In Il. 18. 486-489 heißt es mit fast denselben Worten:

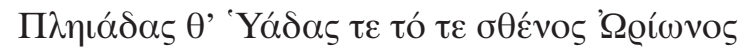

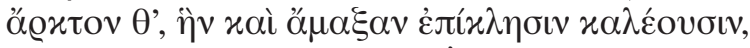

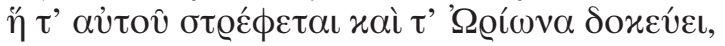

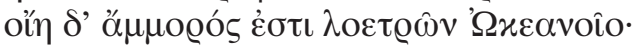

${ }^{5}$ Od. 5. 271-275. 
Eudoxos von Knidos, einer der bedeutendsten Mathematiker und Astronomen der Antike (400 bis ca. 347), nennt den Bootes erstmals Arktophylax (frg. 24) neben der mehrfachen Erwähnung der Bären [z. B. frgg. 15, 28, 29 (Großer Bär), 33 (Kleiner Bär)]. Eine speziell astronomische Darstellung des Großen Bären findet sich in den Phainomena des Arat von Soloi aus dem dritten Jahrhundert v. Chr. (92-96):

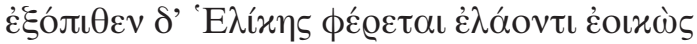

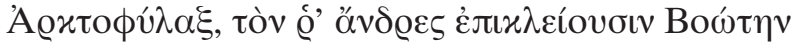

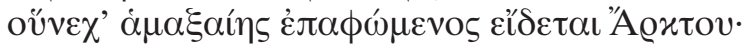

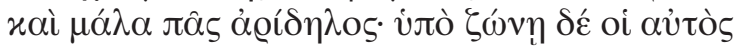

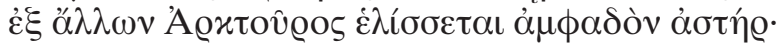

In diesem Zusammenhang fällt insbesondere die dreifache Bezeichnung des Großen

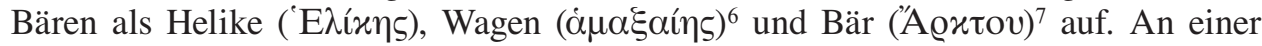
weiteren Stelle (25-44) kommt der Dichter speziell auf die beiden Bären, ihre Gestalt, ihre unterschiedliche Bezeichnung und den zugrunde liegenden Mythos zu sprechen. ${ }^{8}$ Darüber hinaus steht auch Theokrit (24. 11) als Zeuge dieser Tradition.

\footnotetext{
zu verstehen.

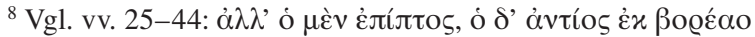

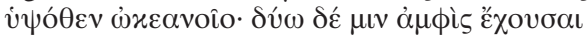

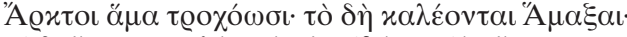

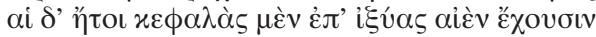

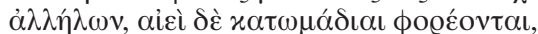

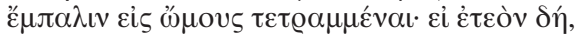

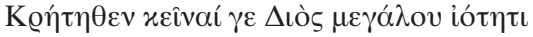

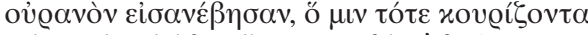

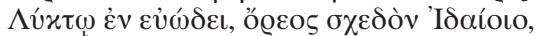

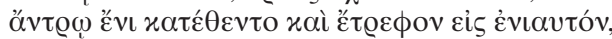

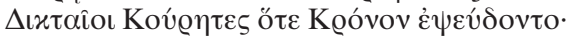

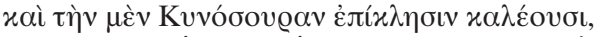

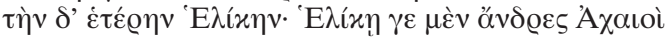

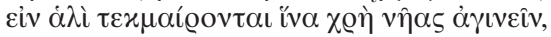

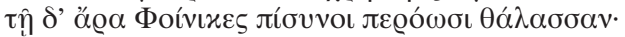

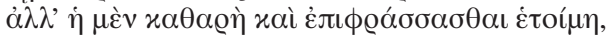

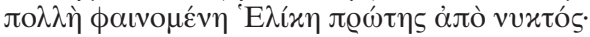

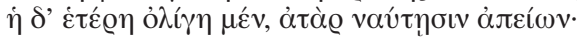

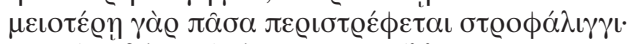

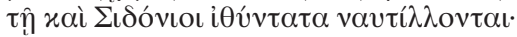

${ }^{6}$ Bei Arat $94 \dot{\alpha} \mu \alpha \xi \alpha i ́ \eta \varsigma$ 'A@xтov handelt es sich um den Versuch, verschiedene Nomenklaturen zu vereinen und insbesondere auch die Etymologie von ö $\mu \alpha \alpha$ $\alpha \xi \omega v$ zu erklären: Die Bärinnen scheinen die

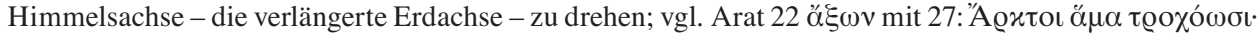

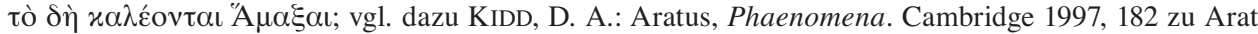

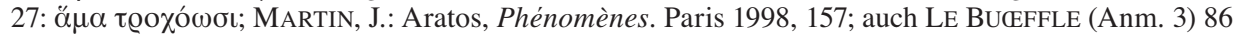

${ }^{7}$ Arat bezeichnet das Sternbild an dieser Stelle als Wagen-Bär, um dieses von dem des Kleinen Bä-

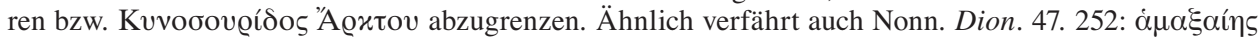

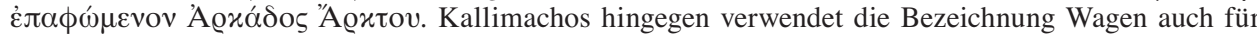

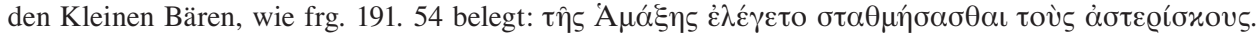

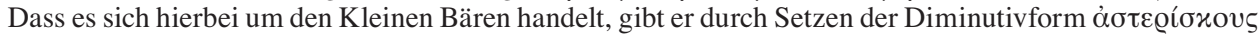




\section{Lateinische Vorbilder}

In der lateinischen Literatur schreibt erstmals Germanicus in seiner Aratea über die Große Bärin in Verbindung mit dem Bootes ${ }^{9}$ und kommt explizit auf ihre verschiedenen ${ }^{10}$ Bezeichnungen zu sprechen. ${ }^{11}$

Auch Manilius erwähnt die Formation des Großen Bären (maior Helice), wobei für ihn jedoch deren Charakter als Schiffersternbild im Vordergrund steht (1. 296-298):

maioremque Helice maior decircinat arcum

(septem illam stellae certantes lumine signant)

qua duce per fluctus Graiae dant vela carinae.

Die Gleichsetzung des Großen Bären ${ }^{12}$ mit dem Wagen belegt außerdem eine Erläuterung aus der lateinischen Übersetzung der Phainomena des Arat aus dem vierten nachchristlichen Jahrhundert. Rufus Festus Avienus schreibt dort über die Doppelbenennung des Sternbildes (vv. 103-104): ${ }^{13}$

Voce feras Ursasque et Plaustra vocare solemus

fabula namque Ursas, species dat Plaustra videri.

Statius Thebais vv. 692-693

Wie unschwer zu erkennen, konnte Statius bei der Konzeption der Passage sehr wohl auf eine lange Tradition zurückgreifen, wenn er schreibt:

Sed iam temone supino

languet Hyperboreae glacialis portitor Ursae.

${ }_{9}^{9}$ Den Bootes bezeichnete bereits Cicero (frg. 16. 1) als Arktophylax. Obwohl beide Namen belegt sind, ist die Form Bootes häufiger. Weitere Bezeichnungen sind Custos (Vitr. 9. 4. 1), Custos Ursae (Ov. fast. 2. 153), Icarus (Prop. 2. 33. 24; Ov. met. 10. 450). Zu den Bärinnen auch Cic. frgg. 5-7.

${ }^{10}$ In der lateinischen Literatur begegnen die Begriffe plaustrum, currus und temo (Schol. Arat 27 Usener) gleichwertig. Meist wurde jedoch der Große Bär von den Römern als Septemtriones, den sieben Dreschochsen, die wie auf einer Tenne am Himmel im Kreis umhergehen, wahrgenommen; vgl. Varro bei Gell. 2. 21. Eine andere Erklärung bietet Avienus: quod ita sunt septem stellae sitae, ut ternae proximae quaeque efficiant tria trigona. Zur Namensgebung vgl. auch GUNDEL, W.: De stellarum appellatione et religione Romana. Gießen 1907, 160-161.

${ }^{11}$ Vgl. Germ. 24-47. Dazu Maurach, G.: Germanicus und sein Arat. Heidelberg 1978, 36-46.

12 Auf die Bären kommt Avienus explizit in vv. 99-137 zu sprechen - Cic. frgg. 5-7 und Germ. 24-47 liefern neben Arat die Vorlage. Vgl. dazu WeBER, D.: Aviens Phaenomena, eine Arat-Bearbeitung aus der lateinischen Spätantike. Untersuchung zu ausgewählten Partien. Diss. Wien 1986, 68-80.

${ }^{13}$ Neben dieser bezeichnenden Stelle finden sich Verweise auf die Bären und die Gleichsetzung des Großen Bären mit dem Wagen in vv. 143, 261, 355, 443, 446, 509, 1230. Die Bezeichnung Helice für Ursa maior findet sich in vv. 122, 124, 144, 158, 260, 379, 413. 
Obgleich die vorliegende Wendung auf den ersten Blick recht klar zu verstehen ist, zeigen sich auf den zweiten Blick dennoch einige Schwierigkeiten, die es im Detail zu klären gilt.

Der Dichter spricht an dieser Stelle vom portitor glacialis, dem eisigen Träger, der, wie Hermann Heuvel in seinem Kommentar zum ersten Buch der Thebais erkannt haben möchte, mit dem Sternbild des Bootes gleichzusetzen ist, ${ }^{14}$ der als Treiber und Lenker des Wagen-Bärs ${ }^{15}$ auftritt. Bei genauerer Betrachtung jedoch muss diese Identifikation angezweifelt werden. Ein Problem zeigt sich nämlich darin, dass portitor niemals synonym für auriga, agitator (Wagenlenker) oder heniochus (Fuhrmann) verwendet wird. ${ }^{16}$

Als Substantiv heißt portitor zunächst einmal ,der Träger', so wie das Sternbild des Wassermanns sein Gefäß (Anth. 677. 4 portitor urceoli) und der Widder Helle, die Schwester des Phrixus, trägt (Colum. 10. 155 portitor Helles; Lucan. 4. 57: delapsae portitor Helles; Mart. 9. 71. 7: portitor Helles). Die Identifikation von portitor mit Bootes erscheint somit nicht gegeben.

Den Anstoß für eine wahrscheinlich richtigere Deutung bietet eine Anmerkung von D. R. Shackleton Bailey in seiner zweisprachigen Statius-Ausgabe (zur Stelle 1. 93): ${ }^{17}$ „,...) Probably thinking of Bootes, Statius substitutes portitor for Lucan's plaustrum." Die Wendung, auf die Bailey anspielt, findet sich an einer Stelle in der Pharsalia des Lucan. In 2. 722 benennt dieser dort den Wagen des Bootes als plaustra (Et iam Plias hebet, flexi iam plaustra Bootae). In Verbindung mit der von Bailey zwar nicht mehr erwähnten Stelle 5. 23, in der Lucan ,vom eisigen Wagen der hyperboräischen Bärin' (Nam vel Hyperboreae plaustrum glaciale sub Ursae) spricht, entsteht der Bezug zum Sternbild des Großen Bären. ${ }^{18}$

Gleichwohl Bailey zu Unrecht in der Statius' Passage einen direkten Bezug zu Bootes erkennt, geht er dennoch richtig davon aus, dass portitor von Statius für das plaustrum bei Lucan gesetzt worden ist. Und diese Erklärung erscheint $\mathrm{m}$. E. aus einem gewichtigen Grund richtig. Geht man davon aus, dass das Sternbild Großer Bär das Bild des Wagens mit einschließt, somit der Wagen ein Teilsternbild (Asterismus) des Großen Bären ist, nimmt der Große Wagen den unteren Teil des gesamten Stern-

14 Vgl. dazu Heuvel, H.: Publii Papinii Statii Thebaidos liber primus ... commentario ... exegetico instructus. Diss. Groningen-Zutphen 1932, ad 1.

${ }^{15}$ Den Zusammenhang zwischen dem Arktophylax in Form des Arktur und dem Wagen stellt Eratosthenes in der Erigone deutlich her. In seiner Darstellung ist die Große Bärin der Wagen, mit dem Ikar(i)os, der Vater der Erigone, den Menschen den Wein bringt; vgl. dazu RosoKOKI, A.: Die Erigone des Eratosthenes. Eine kommentierte Ausgabe der Fragmente. Heidelberg 1995.

${ }^{16}$ Vgl. dazu ThLL vol. X 2 c. 41-43 s.v. portitor. Dort heißt es zu der hier erwähnten Stelle bei Statius in aller Ausführlichkeit: „,sc. Bootes ursam plaustro vehens? Ad confusionem imaginum cf. Sen. Herc. O 1523 sub plaustro ... ursae; gubernatorem intelligit Heuvel, 1932, ad 1.; circitor coni. Alton, Class. Quart. 17 (1923) 175sqq.; inepte SCHOL.: Orphiuchum significat.“

${ }^{17}$ Vgl. Shackleton Bailey, D. R.: Statius, Thebaid and Achilleid. Vol. I. Cambridge-London 2003, 90, Anm. 71.

${ }^{18}$ Obgleich der Bezug von glacialis als Genitiv zu Ursae sachlich naheliegt, sollte er aus sprachlicher Sicht jedoch ausgeschlossen werden. Insbesondere der Genitiv Hyperboreae und die Verwendung von temo in Verbindung mit ursa weisen eindeutig auf den Großen Bären hin, da die Deichsel des Kleinen Bären in der Antike als Hundeschwanz (Cynosura) gedeutet und dementsprechend bezeichnet worden ist. 
bildes ein. Aus der bildlichen Darstellung selbst kann gefolgert werden, dass die Bärin als Ganzes vom Großen Wagen gewissermaßen getragen wird. Somit drückt die bei Statius verwendete Bezeichnung portitor glacialis Hyperboreae ursae nichts anderes aus als die doppelte Funktion desselben Sternbildes.

Der erste Fragenkomplex lässt sich aus den erbrachten Ergebnissen somit leicht beantworten. Wie gezeigt worden ist, konnte Statius auf eine durchaus lange literarische Tradition zurückgreifen, die sich explizit mit den Sternbildern Bootes, welcher hier ausgeschlossen werden konnte, und Großer Bär auseinandergesetzt hat. Das konkrete Vorbild für die sprachliche Konzeption der Passage dürfte in Lucans Pharsalia zu sehen sein, an dem sich Statius augenscheinlich orientiert hat.

Inwiefern jedoch, so lautet der zweite Fragenkomplex, kann aus Statius' astronomischen Angaben des verblassenden Wagen-Bärs ein etwaiger Zeitpunkt erschlossen werden, zu dem der Dichter das Fest im Palast des Adrastos hat stattfinden lassen wollen? Ist es möglich, dass Statius bei der Konzeption der Passage eigene Beobachtungen des Sternenhimmels in Rom einfließen ließ, ergo das mythische Fest des Adrastos in das real existierende Rom der Jahre zwischen 80 und 94 n. Chr. transferiert worden ist? Eine Antwort darauf zu geben, ist Ziel des zweiten Teils der Studie.

\section{ASTRONOMISCHE DEUTUNG}

In Statius' Konzeption der Passage lässt der Dichter auf das Ermatten des Sternbildes hinweisen, womit grundsätzlich das Fortschreiten der Nacht bezeichnet werden soll. ${ }^{19}$ Darauf verweisen beispielhaft Margarethe Billerbeck ${ }^{20}$ im Hinblick auf v. 131 von Senecas Hercules furens - ,verso temone weist auf die Stunden nach Mitternacht, wenn der Wagen auf seiner Himmelsbahn den höchsten Punkt erreicht hat und Bootes die Deichsel zur Fahrt nach unten wendet" - als auch implizit Michael Hillen ${ }^{21}$ ad locum - „[D]ie Deichsel weist schräg nach unten und somit auch der ganze Wagen, dessen Richtung nun nicht mehr geändert werden kann.“

Allerdings muss es sich hierbei nicht zwangsläufig um den kosmischen Untergang $^{22}$ bzw. acronychischen Aufgang ${ }^{23}$ eines bestimmten Sternes handeln, wie es für das bereits angesprochene, doch ausgeschlossene Sternbild des Bootes gilt. Es scheint

\footnotetext{
${ }^{19}$ Nach der Stellung der in jenen Breiten immer sichtbaren Bärinnen bestimmte man in der Antike die Nachtzeit; vgl. dazu HüBner, W.: Die Rezeption der Phainomena Arats in der lateinischen Literatur. In Horster, M. - REITZ, C. (Hgg.): Wissensvermittlung in dichterischer Gestalt. Stuttgart 2005, $133-154$.

${ }^{20}$ Seneca, Hercules Furens. Einleitung, Text, Übersetzung und Kommentar von M. BILLERBECK. Leiden 1999, 248.

${ }^{21}$ Hillen, M.: Studien zur Dichtersprache Senecas: Abundanz, explikativer Ablativ, Hypallage. Berlin 1989, 117.

${ }^{22}$ Unter der Bezeichnung, kosmischer Untergang‘ versteht man das Untergehen eines Sterns bzw. Sternbildes zum Zeitpunkt des Sonnenaufgangs. Vgl. dazu Denningmann, S.: Die astrologische Lehre der Doryphorie. Eine soziomorphe Metapher in der antiken Planetenastrologie. München-Leipzig 2005.

${ }^{23}$ Unter , acronychischer Aufgang' ist das Aufgehen eines Sterns bzw. Sternbildes zum Zeitpunkt des Sonnenuntergangs zu verstehen.
} 
vielmehr möglich, dass die Verbform languet ${ }^{24}$ in ihrer Bedeutung auf das allmähliche Verblassen bzw. Verdunkeln der hellsten Sterne - Alioth und Alkaid - des Sternbildes hinweist, wie dies bei beginnendem Sonnenaufgang im Nordwesten zu beobachten ist. So ist es möglich, dass auch das ,Verblassen“ des Wagen-Bärs, der aufgrund seiner Lage in der Nähe des nördlichen Himmelspols als Zirkumpolarstern für die Beobachter von der Breite des Mittelmeeres aus zu keiner Zeit des Jahres untergeht, ${ }^{25}$ sondern ganzjährig um den Polarstern kreist, beobachtet werden kann. ${ }^{26}$ Vor diesem Hintergrund scheint es durchaus möglich, in Statius' Angabe ein mögliches Datum des fiktiven Festes im Palast des Adrastos erschließen zu können.

Geht man für die Abfassung der Thebais von einem Zeitraum zwischen 80 und 92 n. Chr. - in der heutigen Forschung als wahrscheinlich angenommen ${ }^{27}$ - und als Ort der Abfassung Rom aus, dann lassen sich mit Hilfe der frei zugänglichen PC Programme ,Planetary, Lunar and Stellar Visibillity 3.0.1' (PLSV) und ,Stellarium 0.10.5` präzise Daten für die letzte Sichtbarkeit des Wagen-Bärs vor Sonnenaufgang ermitteln.

Bei der Verwendung stellt sich schnell heraus, dass gerade in den Frühjahrsmonaten (März bis Mai), in denen das Sternbild selbst vor Sonnenaufgang am Nordwest-Himmel zu sichten ist, das Verdunkeln der Sterne weniger eindrucksvoll erscheint als in den Herbstmonaten September bis November. In dieser Zeitspanne befindet sich der Wagen-Bär auf seiner zirkumpolaren Bahn bei Sonnenaufgang deutlich näher dem Osten, was den Eindruck des allmählichen Verblassens der Sterne für einen Beobachter markanter erscheinen lässt als im Frühjahr. Betrachtet man vor diesem Hintergrund den Zeitraum September bis November der Jahre 80, 85, 90 und 92 n. Chr. näher, ${ }^{28}$ ergeben sich folgende Ergebnisse.

Von September bis November der Jahre zwischen 80 und 92 n. Chr. war auf der geographischen Breite von Rom (41 $\left.{ }^{\circ} 52^{\prime \prime} 48^{\prime}\right)$ das Verblassen bzw. Verdunkeln von Alioth und Alkaid als hellsten Sternen des Wagen-Bärs von ca. 5:24 Uhr (1. September) bis ca. 7:24 Uhr (1. Dezember) in der Zeit der beginnenden Dämmerung bis zum endgültigen Sonnenaufgang zu sehen. Der mögliche Zeitpunkt des fiktiven Festes läge somit in einem vagen Zeitraum von vier Monaten.

${ }^{24}$ Vgl. ThLL vol. VII 2 c. $923.75-77$ s.v. langueo; ibid. c. 922. 56-58 s.v. languesco.

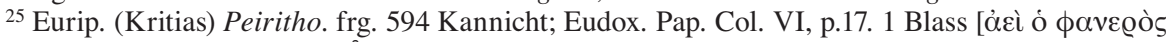

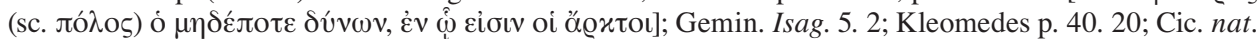
deor. 2. 105; Verg. georg. 1. 246; Hygin. astr. 2. 1 p. 31 Ziré (hoc signum, ut complures dixere, non occidit); Macrob. Somn. 1. 16. 4 ; Achill. Isag. P. 56. 7 Maas; Lact. inst. 7. 14. 8; Isid. nat. rer. 12. 6.

${ }^{26}$ Eine Erklärung, warum die Bärinnen nicht im Meer versinken (d. h. unter den Horizont gehen), bietet Ovid met. 2. 508-530 ausführlich; Statius (Theb. 7. 8-9) berührt diesen Gedanken ebenfalls.

${ }^{27}$ Shackleton Bailey (Anm. 17) 2-3 plädiert u. a. für eine Abfassungszeit zwischen 80 und $92 \mathrm{n}$. Chr.

${ }^{28}$ Da sich die Ergebnisse innerhalb der zu betrachtenden 14 Jahre jährlich nur um wenige Minuten unterscheiden, habe ich mich auf den Auf- und Untergang der Jahre 80, 85, 90 und $92 \mathrm{n}$. Chr. beschränkt. 
Aus Statius' früheren Angaben in den Versen 350-355 könnte dieser Zeitraum jedoch deutlich eingeschränkt werden. Dort heißt es:

\section{Iam claustra rigentis \\ Aeoliae percussa sonant, venturaque rauco \\ Ore minatur hiems,}

Der Dichter spricht an dieser Stelle explizit vom Winter (hiems) bzw. einem Wintersturm, der mit rauer Stimme sein Kommen (ventura) androht. Der Hinweis auf dessen noch bevorstehendes Eintreten (ventura) lässt auf einen Zeitraum schließen, der ungefähr zwischen September und vor Dezember liegt. Leicht ist es jedoch, diesen Zeitraum noch weiter zu verkleinern, wenn man sich vor Augen hält, dass in den genannten Jahren in der Zeit zwischen November und Dezember der Wagen-Bär während der Dämmerung und des vollständigen Sonnenaufgangs im Nordwesten stand, so dass das Verblassen der Sterne bei Sonnenaufgang wohl weniger eindrucksvoll erschien.

Geht man andererseits davon aus, dass der Winter zwar bevorstand, aber noch nicht eingetreten war, wird ein Zeitraum zwischen Ende September und Ende Oktober wahrscheinlicher als im November. Zu dieser Zeit lag die letzte Sichtbarkeit der hellsten Sterne zwischen 5:56 Uhr und 6:40 Uhr.

Für die Bestimmung eines Datums des fiktiven Festes zu Ehren Apolls können diese Ergebnisse dementsprechend hilfreich sein: Sollte Statius bei der Konzeption der untersuchten Passage nicht nur allgemein metaphorisch auf das Fortschreiten der Nacht hingewiesen haben wollen, sondern ganz unterschwellig astronomische Phänomene seiner eigenen Zeit aufgenommen haben, um eine innere chronologische Abfolge der dargestellten Ereignisse zu gewährleisten, dürfte der Dichter den Termin des Festes bei Adrastos in den Spätherbst gelegt haben, wohl konkret in den Zeitraum zwischen Ende September und Ende Oktober. Einen anderen Zeitpunkt ließe die astronomische Analyse nicht zu.

Diese Vermutung scheint sich im Übrigen mit dem Verweis auf die düstere Grundstimmung ${ }^{29}$ des Epos selbst zu manifestieren, dessen innere chronologische Einordung in die Dunkelheit des fortschreitenden Spätherbstes besser passt als in den Beginn des Frühsommers.

${ }^{29}$ Vgl. u. a. KRUMBholz, G.: Der Erzählungsstil in der Thebais des Statius. II: Die Wesenszüge des Stils. Glotta 34.3-4 (1955) 237: ,Vorwiegend solche Nachtbilder von unheimlicher Atmosphäre geben dem ganzen Werk jenen eigentümlichen düsteren Aspekt." THOME, G.: Vorstellungen vom Bösen in der lateinischen Literatur. Stuttgart 1993, 307, Anm. 776: ,[V] ]on der Grundstimmung einer Welt der Dunkelheit, die die Thebais durchzieht, scheinen selbst Gestalten wie Adrast und Argia tangiert (...).“ 
Fazit

Das Ergebnis dieser kurzen Studie zeigt, dass gerade an der untersuchten Stelle der Verweis auf ein astronomisches Phänomen nicht nur grundsätzlich als lyrischer Topos erfolgt sein könnte, sondern reflektiert und bewusst eingefügt worden ist. Statius hat bei der Konzeption der Passage möglicherweise das langsame Verblassen bzw. Verdunkeln der hellsten Sterne des Wagen-Bären vor Augen gehabt, das er in die Thebais einfließen ließ.

Ob in letzter Konsequenz generell hinter Statius' astronomischen Angaben stets weniger tradierte Topoi als vielmehr reflektierte und bewusst gewählte Überlegungen zu sehen sein sollten, scheint möglich. Den letzten Beweis muss diese Untersuchung jedoch schuldig bleiben, würde er doch das primäre Ziel und den Rahmen dieser kurzen Studie überschreiten.

Boris Hogenmüller

Institut für Altertumswissenschaften

Julius-Maximilians-Universität Würzburg

Deutschland

boris.hogenmueller@gmail.com 